
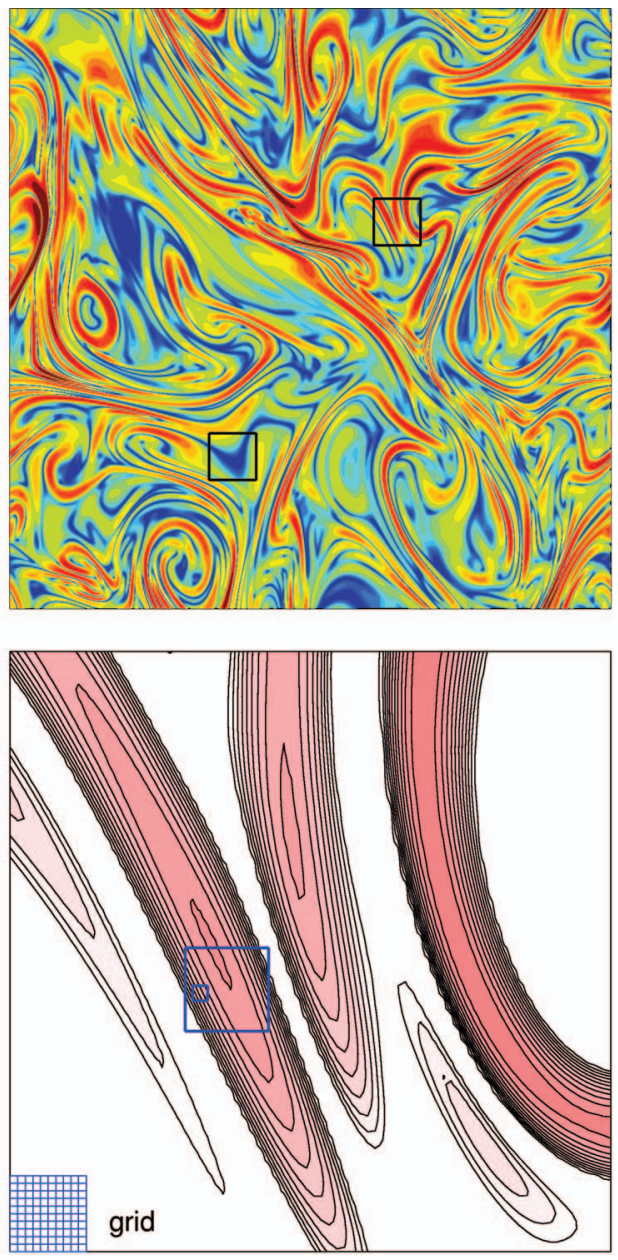

\section{Scalar dissipation fronts in high-Schmidt number mixing}

\section{Jörg Schumacher}

Fachbereich Physik, Philipps-Universität Marburg, D-35032

Marburg, Germany

Herwig Zilken

Visualization Laboratory, Zentralinstitut für angewandte

Mathematik, Forschungszentrum Jülich, D-52425 Jülich,

Germany

Bruno Eckhardt

Fachbereich Physik, Philipps-Universität Marburg, D-35032

Marburg, Germany

Katepalli R. Sreenivasan

International Centre for Theoretical Physics, 34014 Trieste, Italy

(Received 27 September 2005;

published online 30 December 2005)

[DOI: $10.1063 / 1.2130687]$

Passive scalar fields advected by turbulent flows develop regions of very strong gradients. Such regions are of interest because of their relation to dissipation, mixing, and chemical reactions. The upper left panel of Fig. 1 shows a twodimensional cut of the instantaneous scalar dissipation rate (proportional to the gradient squared) obtained in a direct numerical simulation. The Schmidt number is 32, i.e., the Batchelor scale $\eta_{B}$ of the passive scalar is about a sixth of the Kolmogorov scale $\eta$ of the advecting fluid. The color
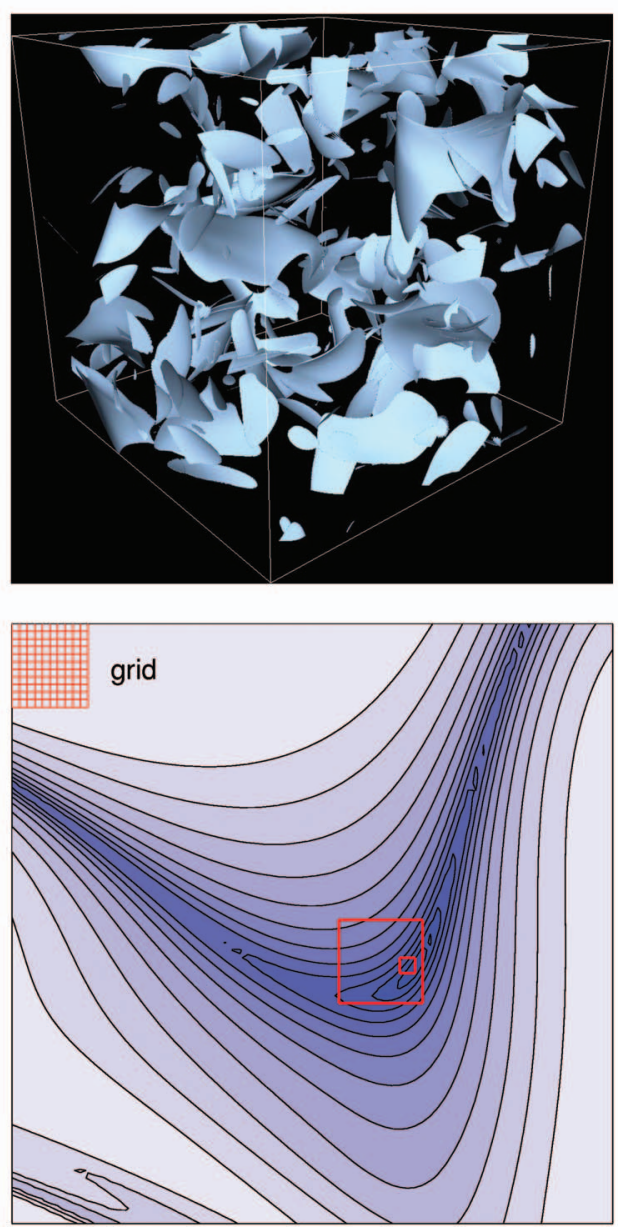

coding runs logarithmically from $10^{-5}$ (blue) to $10^{2}$ (red) in units of the mean scalar dissipation rate. ${ }^{1}$

Magnifications of the of the two framed regions are given in the lower panels. They show that many structures are much narrower than the mean scales $\eta$ and $\eta_{B}$, which are indicated by the colored squares. Hence much higher resolutions for a proper numerical representation are required. The grid resolution is $N=1024$ in all three directions and also indicated by small grids in both panels.

In three dimensions, the high intensity regions of the scalar dissipation rate are concentrated in sheets. This is evident from the isosurfaces of the field at a level of 11 times the mean scalar dissipation value. This is shown in the upper right panel. Their geometric properties enter models that describe the small-scale intermittency of passive scalar turbulence or the intensity of chemical reactions in nonpremixed turbulent combustion. ${ }^{2}$

Computations were done on $256 \mathrm{CPUs}$ of the IBMJUMP cluster at the John von Neumann-Institute for Computing in Jülich (Germany). Support by Deutsche Forschungsgemeinschaft (DFG), and the US National Science Foundation (NSF) is gratefully acknowledged.

${ }^{1}$ J. Schumacher and K. R. Sreenivasan, Phys. Rev. Lett. 91, 174501 (2003). ${ }^{2}$ J. Schumacher, K. R. Sreenivasan, and P. K. Yeung, J. Fluid Mech. 531, 113 (2005). 